\title{
Sustainable Cycle-Oriented Society from the Viewpoint of Diversity in Consumers' Decision Making
}

\author{
Takeshi Maeda and Toshiharu Taura \\ Graduate School of Science and Technology, Kobe University \\ E-mail:maeda@ma-5.scitec.kobe-u.ac.jp,Taura@mech.kobe-u.ac.jp
}

\begin{abstract}
An approach to change the present cycle-oriented society is needed. Such a society has progressed in a direction that mainly restricts consumer behavior, such as by promotional and legal restrictions, in a closed loop of product circulation. We proposed the hypothesis that an effective approach to developing a recycling society is to diversify consumers' behavior. We considered the support of the behavior information at the time of product disposal as a method of social control of the diversity of consumer behavior. We verified this hypothesis by conducting simulations using different social scenario models. We also analyzed the control conditions of behavior diversity. As a result, we determined that an increase in behavior diversity could become effective for cycleoriented society development.
\end{abstract}

Key words: diversity, consumer behavior, social control, product disposal

\section{Introduction}

In recent years, the effective use of resources and energy and the control of environmental problems have become important issues in societal development. Therefore, the shift of a social system to a sustainable cycle-oriented society is needed to deal with these issues, and much research is in progress to realize that aim. Management design that foresees the entire circulation process of a product from design to recovery[1], and the legal maintenance of resource recycling are typical approaches. The basic concept is that product circulation is aimed at a one-way closed loop through such a method. However, such methods restrict consumers' behaviors at the time of product disposal, and it can be said that this decreases flexibility. It can be said that strong social regulation and restriction are problematic and may also give rise to new problems such as illegal disposal, when building a self-reliant sustainable society. In this research, we apply the consumer's decision-making behavior, what is conventionally considered to focus on the time of purchase, at the time from use to the disposal of a product as well. We propose the hypothesis that increasing the flexibility of consumer behavior at the time of product disposal and diversifying this behavior can also lead to a sustainable cycle-oriented society.

\section{Research on concept of diversity}

We decided to consider the social influence of the diversification of behavior at the time of product disposal, focusing on the diversity of consumer behavior, as a method of hypothetical embodiment. The concept of diversity is said to be as important as the diversity and heredity diversity of species for the maintenance of evolution and a life mechanism. Moreover, many researchers have carried out similar research on the relationship between the instability of the unemployment rate and economy, by examining the diversity of industry and the classification of occupations[2], or on the relationship between the diversity in industry and the stability and development of regional economies in social formation[3].

We apply the concept of this diversity to consumer behavior at the time from use to the disposal of a product in this research. We analyze the influences on sustainable cycle-oriented society formation, such as the diversity of consumer behavior, the final disposal rate, and the stability of behavior, by social simulation of disposal behavior. We explore the social validity and the conditions of diversification of behavior referring to the results, and verify the hypothesis.

\section{Construction of consumer behavior model}

\subsection{Classification of consumer behavior}

We first consider consumer behavior in the process from the use to the disposal of products. In considering the diversity of the behavior at the time of disposal, we classify consumers' behavior when consumers consider the disposal of a product, according to the continuation of possession of a product, the whole or partial change in the 
product mechanism, and the actual component of the behavior (Tab.1).

Tab.1: Behavior classification at the time of product disposal

\begin{tabular}{|c|c|c|}
\hline & \multicolumn{2}{|c|}{ Components of behavior } \\
\hline \multirow{6}{*}{$\begin{array}{l}\text { Continuation } \\
\text { of possession } \\
\text { of product }\end{array}$} & \multicolumn{2}{|c|}{ Possession continuation } \\
\hline & \multicolumn{2}{|c|}{ Functional addition, upgrade } \\
\hline & \multirow{2}{*}{ Repair } & Contractor repair \\
\hline & & Repair by oneself \\
\hline & \multicolumn{2}{|c|}{ Different use from basic purpose } \\
\hline & \multicolumn{2}{|c|}{ Inclusion of other products } \\
\hline \multirow{5}{*}{$\begin{array}{l}\text { Disposal of } \\
\text { product }\end{array}$} & \multirow{2}{*}{$\begin{array}{c}\text { Conversion to } \\
\text { economic } \\
\text { value }\end{array}$} & Contractor trade-in \\
\hline & & $\begin{array}{l}\text { Dealing between individu- } \\
\text { als }\end{array}$ \\
\hline & \multicolumn{2}{|c|}{ Contribution, transfer } \\
\hline & \multirow[t]{2}{*}{ Final disposal } & $\begin{array}{l}\text { Disposal by regulation } \\
\text { method } \\
\text { - Regulation disposal } \\
\text { - Recycling recovery }\end{array}$ \\
\hline & & Illegal disposal \\
\hline
\end{tabular}

\subsection{Flow of behavior from product use to dis- posal}

Next, we consider the flow of behavior from product use to disposal. When we regard abandonment behavior as a decision-making problem, we can assume that a consumer acts on disposal taking into consideration consumption and the environment factor of the value upon the use of a product. It can be said that such a flow strongly resembles the flow of purchase shown by the EBM model[4], which is one of the typical conceptual models of purchase behavior. Then, we applied the EBM model extended to the time from the use to the disposal of a product, and modeled the flow of consumer behavior at the time of product disposal consideration (Fig.1).

We used the Fishbein model by making the alternative plan evaluation before disposal into a multi attribute decision-making problem. As for the attributes that a consumer uses for decision-making, we examined each element considered to be important for disposal behavior. They are "economical efficiency”, "time and effort”, “environmental impact”, and "product possession intention”. We considered two products, a desktop PC and a bicycle, for which the fundamental final disposal behaviors differ.

We assigned weight of value to each of these attributes by conjoint analysis, and introduced the type of decisionmaking behavior as the consumer's behavior consciousness index. We conducted conjoint analysis with a choice-based questionnaire concerning the various actions for which each attribute level, such as expense, was changed for the desktop PC and the bicycle. Moreover, since the effective components of action change with the degree of use condition of a product, so we are performing two evaluations that a product has failure or a product has not failure. A questionnaire was distributed on the Internet browser, and effective replies were obtained from 15 college students from September to October 2004 (Tab. 2 ). We assigned weight of value to behavior decisionmaking by the consumer in the simulation, on the basis of the results of the research.

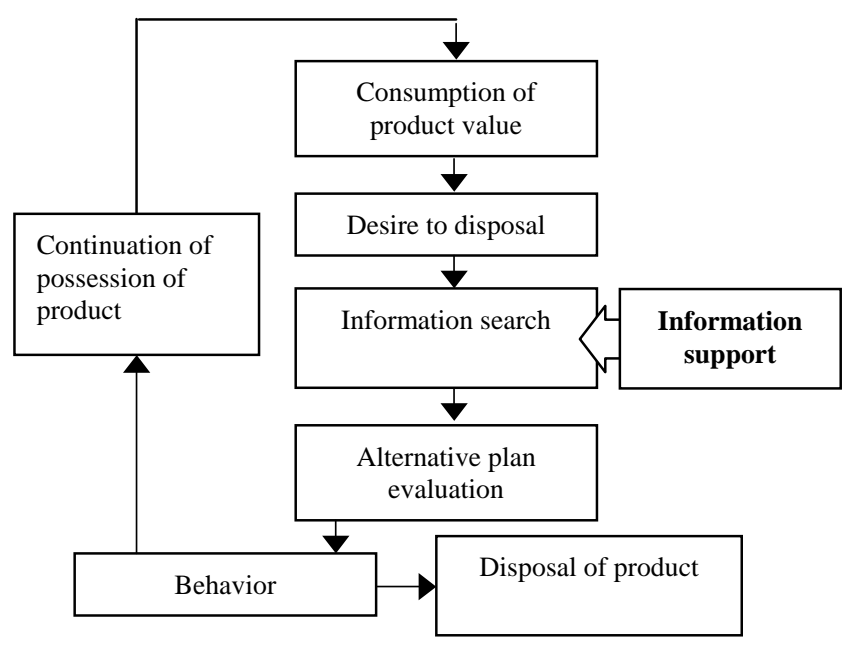

Fig.1: Flow of behavior from use to disposal of product

Tab. 2: Dignity assignment of behavior consciousness by conjoint analysis

\begin{tabular}{|c|c|c|c|c|}
\hline \multirow{2}{*}{$\begin{array}{c}\text { The contents of } \\
\text { an attribute } \\
\text { (One unit) }\end{array}$} & \multicolumn{2}{|c|}{ Desktop PC } & \multicolumn{2}{c|}{ Bicycle } \\
\cline { 2 - 5 } & failure & $\begin{array}{c}\text { With } \\
\text { failure }\end{array}$ & $\begin{array}{c}\text { With no } \\
\text { failure }\end{array}$ & $\begin{array}{c}\text { With } \\
\text { failure }\end{array}$ \\
\hline $\begin{array}{c}\text { Payment value } \\
\text { (¥100) }\end{array}$ & -0.0292 & -0.0652 & -0.1364 & -0.2861 \\
\hline $\begin{array}{c}\text { Conveyance } \\
\text { effort } \\
(1 \mathrm{~km})\end{array}$ & -0.0775 & -0.1209 & -0.2100 & -0.2188 \\
\hline $\begin{array}{c}\text { Required time } \\
(10 \text { min) }\end{array}$ & -0.0969 & -0.0683 & -0.1715 & -0.1044 \\
\hline $\begin{array}{c}\text { Recycling rate } \\
(1 \%)\end{array}$ & 0.0158 & 0.0120 & 0.0185 & 0.0124 \\
\hline $\begin{array}{c}\text { Possession inten- } \\
\text { tion } \\
(0 \text { or } 1)\end{array}$ & -1.5277 & 4.5072 & -2.3261 & 3.6595 \\
\hline
\end{tabular}




\section{Social control for behavior diversification, and diversity evaluation method}

\subsection{Proposal of technique for controlling be- havior diversity}

When we consider the diversity of consumer behavior during product disposal, we must consider the social conditions under which various behaviors exist, and their control. As a social control that can change the diversity of consumer behavior in this study, we propose information support by offering behavior information at the time of disposal as one of our methods. This perusal of the kind and the contents of each behavior that can be carried out when a consumer considers product disposal in database form is defined as information support. The following is this effect. Consumers realize behaviors that they would not recognize by themselves, so they can extend the choice of behavior at the time of disposal consideration. Moreover, the time and effort of behavioral search and execution are alleviated because consumers learn the detailed contents of behavior simply. Thus, it is considered that an indirect increase in action diversity is induced because information support progresses. We also analyzed the influence of information support on behavior diversity in this research.

\subsection{Behavior diversity evaluation using diver- sity index}

Next, we consider the objective evaluation method of the behavior at the time of disposal consideration. In the evaluation of diversity, the Shannon Index of diversity is generally used.

$$
H^{\prime}=-\sum_{i}^{k} p i \times \ln p i
$$

$k$ : The number of kinds of behaviors pi: The share of the i-th kind

We consider the number of kinds of behavior shown in Tab. 1, and the share as the frequency of a behavior to the total occurrence of all behaviors in this research. We evaluate the diversity of consumer behavior by applying this to the diversity valuation plan of the Shannon Index.

\section{Examination by computer simulation}

\subsection{Details of experiment setup}

We model consumers as agents which perform product disposal behavior along with the extended EBM model on a computer, and explore the social influence at the time of indirectly changing the behavior diversity by performing various scenarios for the cognitive state of behavior when a consumer takes disposal into consideration. We also examined a social model by simulation. We used the discrete-event simulation-technologies software Arena.

We first explain the various scenario model setups in this experiment. We used two products, a low-end desktop PC and a standard bicycle. We set up various social scenarios in which the consumer layer was varied by changing the distribution rate of the behavior consciousness of the consumer at the time of disposal behavior decision-making. Various types of diversity and social conditions of diversity were observed. The standard was the action consciousness value drawn from the conjoint analysis conducted for the desktop PC and bicycle, as shown in Tab. 2.

We setup the behavior consciousness distribution layer of the consumer in alignment with the normal distribution which had standard deviations relative to the standard value of $10 \%, 20 \%, 40 \%, 80 \%$, and $160 \%$, and expressed the difference in the consumer layer by five social scenario models. Furthermore, we set up various scenarios at the time of changing the cognitive state of the consumer's behavior on the basis of social control conditions. We show the contents as follows. Regarding the various behaviors shown in Tab. 1, the continuation of product possession and the final disposal, which are the most fundamental behaviors, are recognized (taken into consideration at the time of alternative plan evaluation) by all agents to have $100 \%$ of probability. We created the scenario from the disposal ratio of the people who recognize various behaviors, by changing the probability of behavior recognition. We created scenarios in which the amount of consumer's behavior knowledge is varied by changing the probability at which the agents recognize behaviors of those other than most fundamental behaviors. We adopted a probability setup of five behavior cognitive rates of $20 \%, 40 \%, 60 \%, 80 \%$, and $100 \%$. Supposing a case where the information support progresses, we further added a scenario at the time of reducing the time level attribute, while the action cognitive rate became $100 \%$.

In this way, we conducted the computer simulation of the model for a total of 60 combinations of scenario.

For the setup of the final disposal behavior for each product, we considered free regulation disposal as the final disposal behavior for the bicycle, and charged recycling and illegal disposal as final disposal behavior for the desktop PC.

In this experiment, we treat the number of steps as a time axis, and we analyzed the consumer behavior at the time of disposal consideration from the diversity index and the rate of each final disposal behavior during the 3600 steps (about ten years) from 3600 steps to 7200 steps, where the period from 0 steps to 3600 steps was 
that required for product circulation to become stable. The diversity index and the rate of final disposal, which were obtained in this experiment, were computed from the occurrence of each behavior and the total occurrence of all behaviors of the consumer agents during the 3600 steps.

\subsection{Consideration of experimental result}

We plotted the diversity of behavior correlated it to the final disposal rate in the 3600 steps for all 60 scenarios first (Fig. 2). The vertical axis indicates the final disposal rate, and the horizontal axis the diversity index of each scenario.

The tendency for the final disposal rate to decrease with increasing diversity index was seen, which indicated the validity of the cycle-oriented society formation that diversification of behavior leads to a decrease of the final disposal behavior.

Next, we examine the stability of each behavior. We divided and computed each occurrence of behavior between the 3600 steps performed every 30 steps, and considered the stability of behavior from the average value of the coefficient of variation in each occurrence. We plotted the vertical axis as the coefficient of variation in the behavior and the horizontal axis as the diversity index in each scenario (Fig. 3).

The coefficient of the variation in behavior decreases with increasing diversity index in Fig. 3. The tendency for behavior to stabilize was seen, and we were also able to show the validity of sustainable society formation through the stabilization of the disposal behavior due to the increase in diversity. From the above experimental result, we were able to validate the hypothesis that the formation of a society with higher cyclicity and stability can be achieved, by aiming at diversification of a product disposal.

Next, we compared and considered the result of below the experiment conducted in this study for each product and scenario, regarding the social situation for increasing the diversity of behavior and the validity of information support proposed by us as an indirect social control by which diversity increases. We show a graph of the transition of a diversity index according to the distribution of a consumer's behavior consciousness for the desktop PC in Fig. 4. The vertical axis indicates the diversity index and the horizontal axis indicates the behavior cognitive rate.

When we examine the difference in a consumer's behavior consciousness distribution deviation, change will seldom be seen in the fundamental transition state. Therefore, we turned our attention to the difference in the behavior cognitive rate. In this case, diversity became the maximum when information support progressed the most. Compared with this, when the behavior cognitive rate was 80\%, diversity was slightly higher.

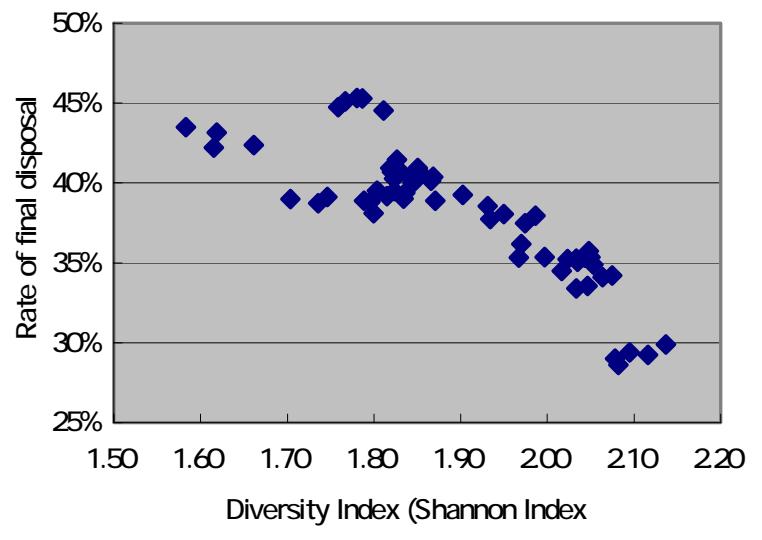

Fig. 2: Diversity and final disposal rate

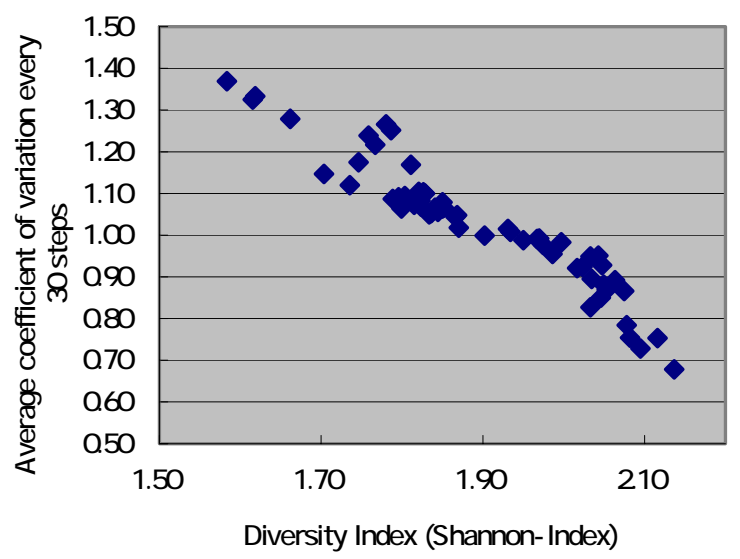

Fig.3: Correlation of diversity and the stability of behavior

In Fig. 5, we show the graph of the transition of the diversity index for a bicycle.

First, we pay attention to the difference in a consumer's action consciousness distribution deviation. When the deviation of consciousness distribution is large and behavior cognitive rate is low, diversity index is low. When the behavior cognitive rate rises, the diversity becomes high compared with the scenario of a small deviation of consciousness distribution. Moreover, when we examine the difference in the behavior cognitive rate, the diversity becomes maximum when the behavior cognitive rate is between $40 \%$ and $60 \%$. If the cognitive rate is higher, diversity falls. We consider that "Winner-Take-All" phenomenon, which indicates that consumers' selections concentrate on a small number of behaviors because it becomes which they can easily recognize, causes diversity to decrease. For this reason, it is possible that it may not always be effective, for increasing the diversity of behavior, to unconditionally advance control with infor- 
mation support. Thus, on the basis of the results of comparing the tendency of scenarios between products, we cannot definitively conclude that information support is effective.

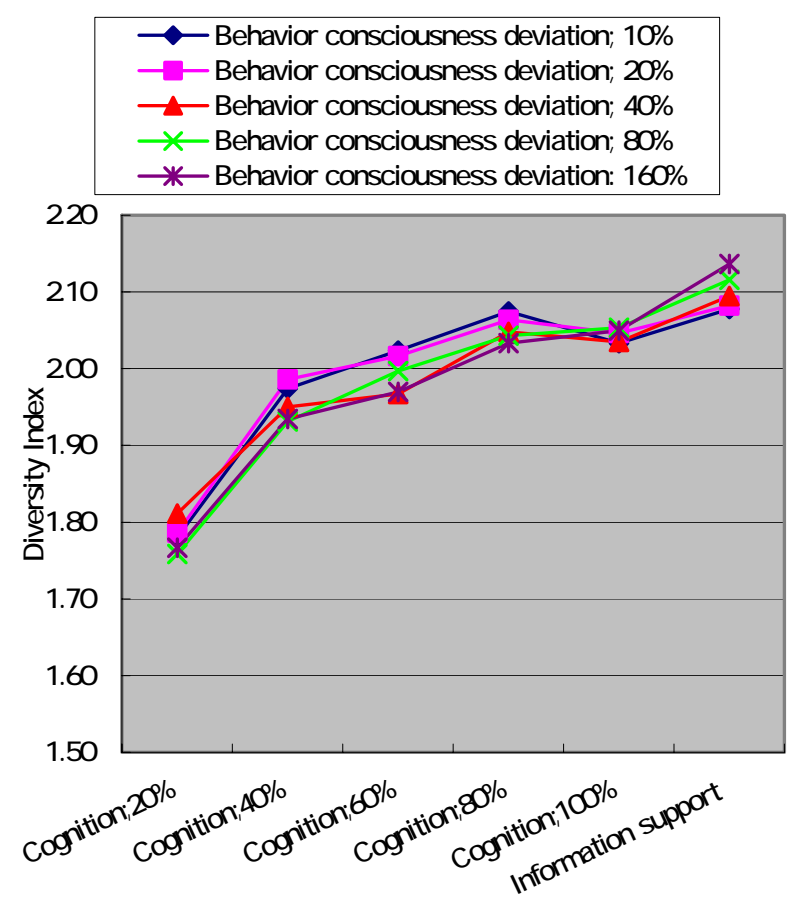

Fig.4: Transition of diversity for desktop PC

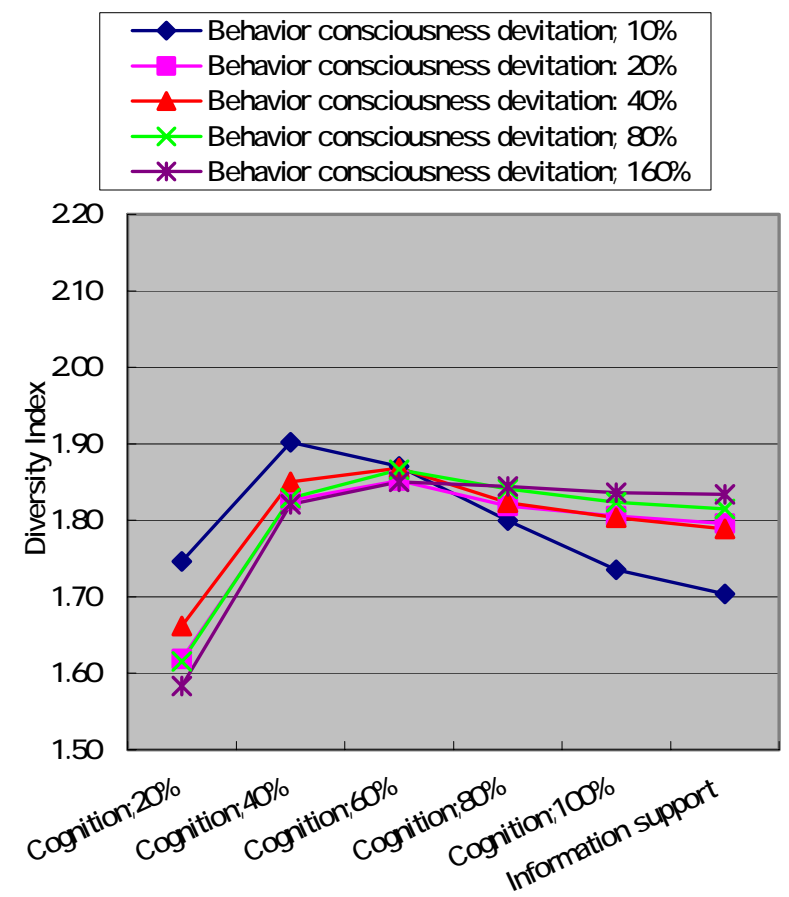

Fig.5: Transition of diversity for bicycle

\section{Conclusion}

In this study, we proposed the hypothesis that an effective approach to the development of a sustainable cycleoriented society is to diversify consumer behavior rather than to restrict it, with regard to product disposal. We validated this hypothesis through computer simulations. As a result, we showed that it could be effective for reducing the number of disposed products, and stabilizing behavior, with the aim of diversifying the behavior at the time of disposal. We also showed that social conditions, including social control with information support proposed as a method for diversifying behavior, differed in the effect depending on the product background. We consider it important to verify which social model best represents the general consumer behavior of actual society and to consider more examples of products when investigating the effective control conditions of the diversification of disposal behavior in recycling society formation.

\section{References}

[1] Y. Umeda: Inverse Manufacturing,; Kogyo Chosakai (1998)

[2] E. E. Malizia, S. Ke: The Influence of Economic Diversity on Unemployment and Stability, Journal of Regional Science, 33, pp. 221-235 (1993)

[3] K. Andou, R. Nakamura: Chiikikeizai no Seichou to Antei, Chiiki Seisaku Kenkyuu vol.13, Nihon Seisaku Toushi Ginkou (2004)

[4] J. F. Engel, R. D. Blackwell and P. W. Miniard: Consumer Behavior, The Dryden Press (1995) 